\title{
Fadiga em pacientes com câncer de mama em tratamento adjuvante
}

\author{
Fatigue in patients with breast cancer in adjuvant treatment
}

Neli Muraki Ishikawa, ${ }^{1}$ Sophie Françoise Mauricette Derchain, ${ }^{2}$ Luiz Claudio Santos Thuler ${ }^{3}$

\section{Resumo}

Em pacientes com câncer em tratamento adjuvante a fadiga é altamente prevalente e é um sintoma debilitante e crônico. 0 objetivo deste estudo foi analisar, na literatura científica, a prevalência e curso da fadiga em pacientes com câncer de mama em tratamento adjuvante. A revisão da literatura foi realizada a partir da base de dados online Pubmed, para o período entre janeiro de 1996 e setembro de 2004, utilizando os seguintes descritores: fatigue e breast cancer. 0 total de artigos obtidos por meio dessa busca foi de 108. Foram identificados 14 artigos que atendiam aos critérios de inclusão para este estudo. A fadiga ocorreu entre 1\% e $94 \%$ dos pacientes com câncer, e foi medida por meio de instrumentos específicos em nove estudos. A fadiga aumentou durante a radioterapia, permaneceu com um platô e retornou ao nível pré-tratamento meses após o seu término. Durante a quimioterapia observou-se que houve aumento da sua prevalência e severidade e que ela é maior durante os primeiros dias após a quimioterapia em relação ao período entre os ciclos. Muitos dos estudos revelaram que a fadiga está significativamente associada a vários fatores físicos, biológicos e comportamentais sendo que quando relacionada ao câncer ela é multidimensional. D evem ser realizadas mais pesquisas controladas, principalmente em mulheres brasileiras com câncer de mama. Estas pesquisas devem avaliar grupos mais homogêneos e com instrumentos válidos e confiáveis para melhor conhecimento da fadiga nesta população e propiciar melhora da qualidade de vida das pacientes.

Palavras-chave: N eoplasias mamárias; Fadiga; Quimioterapia; Radioterapia; Tratamento adjuvante.

\footnotetext{
${ }^{1}$ Fisioterapeuta, M estre em Engenharia Biomédica, Serviço de Integração H umana -Fisioterapia, H ospital do Câncer/Instituto N acional de Câncer/M S 2 Professora do Departamento de Tocoginecologia - Faculdade de Ciências M édicas, Centro de Atenção Integral à Saúde da M ulher CAISM / UNICAMP

3 M édico Epidemiologista do Instituto N acional de Câncer/ Professor adjunto da Universidade Federal do Estado do Rio de Janeiro Endereço para correspondência: N eli M uraki Ishikawa - Serviço de Integração H umana - Fisioterapia, Instituto N acional de Câncer - Rua do Rezende 128, CEP: 20231-092, Rio de Janeiro, RJ. E-mail:nelimuraki@globo.com.
} 


\begin{abstract}
Fatigue is a highly prevalent and a debilitating and chronic symptom in patients with cancer in adjuvant treatment. The objective of this study was to analyze in scientific literature the prevalence and course of fatigue in patients with breast cancer in adjuvant treatment. The literature review was carried through from the on-line Pubmed database between January 1996 and September 2004, using the following key words: "fatigue and breast cancer". The total numbers of article found in this search was 108. Among those, 14 articles had been identified that met the inclusion criteria for this study were identified. The fatigue occurred between $1 \%$ and $94 \%$ in patients with cancer, and was measured by specific instruments in nine studies. The fatigue increased during radiotherapy, remained as a plateau and returned to the pre-treatment level some months after radiotherapy was finished. D uring the chemotherapy it was observed the increase of its prevalence and severity, and the fatigue is higher during the first days after the chemotherapy, when compared to the period between the cycles. M any studies had disclosed that the fatigue is significantly associated with some physical, biological and behavioral facts and, when it is related to cancer it is multidimensional. M ore controlled research must be carried in the future, mainly in Brazilian women with breast cancer. Research with more homogeneous groups and with valid instruments for better knowledge of the fatigue in this population is important in order to improve the quality of life of these patients.
\end{abstract}

Key words. Breast neoplasms; Fatigue; Chemotherapy; Radiotherapy; Adjuvant treatment.

\section{INTRODUÇÃO}

0 câncer de mama é um importante problema de Saúde Pública, devido à sua incidência e mortalidade. No Brasil, de acordo com estimativas realizadas pelo M inistério da Saúde - Instituto N acional de Câncer ${ }^{1}$ (IN CA), no ano de 2005, a taxa bruta de incidência para câncer de mama em mulheres será de 52,93 em 100.000, sendo que o número de casos novos será de 49.470 .

Avanços tecnológicos no diagnóstico e tratamento precoces de câncer têm aumentado a sobrevida das pacientes e conseqüentemente maior ênfase tem sido dada na qualidade de vida dessa população. A qualidade de vida, entre outros aspectos, inclui a fadiga. A fadiga é altamente prevalente e é um sintoma debilitante e crônico em pacientes com câncer sendo observadas nessa população durante e depois de tratamento adjuvante., 2,3 Alguns pacientes sofrem de fadiga por meses ou anos após os tratamentos realizados. ${ }^{4}$

Descrever a fadiga é difícil, e os pacientes se expressam de diversas formas, utilizando termos como se sentindo "cansado, débil, extenuado, esgotado, farto, pesado ou lento". Já os profissionais de saúde empregam termos como astenia, lassitude, prostração, intolerância ao exercício, falta de energia e debilidade. ${ }^{5} \mathrm{~A}$ fadiga pode ser compreendida como subjetiva, afeta a todos, e nos indivíduos saudáveis pode ser um fator de proteção, surgindo como resposta regulatória de estresse físico ou psicológico. Apesar desse efeito protetor, em pacientes com câncer a fadiga freqüentemente é um sintoma desagradável e limitante para a realização de

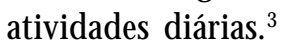

A fadiga está associada com fatores físicos, psicológicos, sociais, cognitivos e comportamentais. A depressão, a incapacidade física, a necessidade de dormir e descansar durante 0 dia e a tendência de atribuir as queixas de fadiga ao tratamento de câncer de mama contribui significativamente para a sua severidade. ${ }^{3}$ As conseqüências do sofrimento produzido pela fadiga levam também à limitação social e diminuição da auto-estima, contribuindo para reduzir a qualidade devida. ${ }^{6}$ A pesar da alta prevalência, a fadiga em pacientes com câncer ainda é pouco entendida. A etiologia da fadiga após o tratamento de câncer ainda permanece incerta. ${ }^{\top}$ Jacobsen et al. propõem que o declínio da hemoglobina durante as repetidas administrações de quimioterapia é acompanhado de aumento na fadiga e declínio na cognição. ${ }^{8}$

0 objetivo desta revisão da literatura é avaliar a prevalência e o curso da fadiga em pacientes com câncer de mama em tratamento adjuvante.

\section{MÉTODO}

A revisão da literatura foi realizada a partir da base de dados on-line Pubmed entre janeiro de 1996 e setembro de 2004, utilizando os seguintes descritores em inglês: fatigue e breast cancer. A revisão foi ampliada por meio de outras fontes como referências citadas nos artigos obtidos. 0 total de artigos obtidos por meio dessa busca foi de 108. 
Para serem selecionados, os artigos deviam preencher os seguintes critérios: ter como foco a fadiga somente em pacientes com câncer de mama, ter sido publicado em inglês e incluir resumo. Foram excluídos os artigos envolvendo pacientes com mais de um ano após o tratamento; estudos qualitativos; artigos de validação de instrumentos; e artigos de intervenção comparativa como pré e pós-tratamento de transplante de medula óssea, regimes de quimioterapia, atividade física, exercícios e intervenção psicológica. 0 total de artigos selecionados atendendo a esses critérios foi de 14.

\section{RESULTADOS}

As 14 publicações etão sumarizadas nos quadros 1 e 2, sendo que 3 estudos são de delineamento transversal ${ }^{9-11}$ e os demais, no total de 11, são de delineamento longitudinal. ${ }^{12-22}$

0 tamanho da amostra analisada nos estudos variou de 14 a 170 pacientes com câncer de mama. A idade das mulheres variou de 22 a 81 anos.

Em seis estudos as pacientes encontravam-se nos estadiamentos I e II, 9,14-16,19 dois estudos informaram somentetratar-se de mulheres em estadiamento inicial, 17,20 dois estudos incluíram mulheres nos estádios I a III,12,18 dois estudos nos estádios I a IV ${ }^{10}$ e em dois estudos não foi informado 0 estadiamento. ${ }^{13,21}$ Apenas um estudo incluiu um grupo controle com mulheres saudáveis. ${ }^{18}$ As populações foram pequenas em dois estudos $\mathrm{s}^{14,16}$ incluindo 14 e 21 mulheres, não sendo possível chegar a conclusões devido ao pequeno número de mulheres estudadas.

As prevalências defadiga encontradas nos dez estudos variaram de $1 \%$ a $94 \%$. A fadiga foi medida por meio de escores em nove estudos e os valores encontrados foram variados conforme apresentado no quadro 1.0 mais freqüente foi o Piper Fatigue Scale, utilizado em quatro estudos. ${ }^{11,14,16,19} \mathrm{~A}$ fadiga também foi avaliada por itens do questionário SF-36 de qualidade de vida em um estudo. ${ }^{17} \mathrm{O}$ coeficiente alfa de Cronbach foi usado para avaliar a confiabilidade dos instrumentos em seis trabal hos e variou de 0,80 a 0,98. 9, 10, 14, 16, 19, 20

Três estudos investigaram a fadiga antes, durante e após a radioterapia. ${ }^{13,15,20} \mathrm{Um}$ avaliou a fadiga antes e durante a radioterapia. ${ }^{17} \mathrm{~A}$ fadiga foi avaliada durante a quimioterapia em seis estudos. ${ }^{9,10,12,16,18,19} \mathrm{H}$ ouve um estudo que avaliou a fadiga após o término de quimioterapia. ${ }^{14} \mathrm{D}$ ois estudaram a fadiga em pacientes que estavam recebendo quimioterapia ou radioterapia. ${ }^{11,22} \mathrm{Um}$ avaliou a fadiga nas mulheres com câncer de mama após tratamento cirúrgico, com quimioterapia e/ ou radioterapia e incluiu também pacientes que não realizaram nenhum tratamento adjuvante; o período de referência para avaliar a fadiga foi 0 dia da cirurgia. ${ }^{22} \mathrm{H}$ ouve aumento da prevalência e severidade da fadiga durante os ciclos de quimioterapia. ${ }^{9}$ A fadiga foi maior nos primeiros dias após a quimioterapia em relação ao período entre os ciclos. ${ }^{16,19}$ $\mathrm{N}$ o estudo com grupo controle as mulheres com câncer de mama em quimioterapia apresentaram significativamente mais fadiga em comparação com 0 grupo de mulheres sem câncer de mama. ${ }^{18}$

Entre os 14 estudos, quatro12, ${ }^{14,18,19}$ forneceram os regimes quimioterápicos utilizados nos tratamentos: ciclofosfamida/methotrexate/fluoracil (CM F); ciclofosfamida, adriamicina e fluoracil (CAF); ciclofosfamida, methotrexate e adriamicina (CMA); ciclofosfamida e adriamicina (CA); adriamicina (A). Foram também observadas maiores taxas de fadiga em mulheres que receberam adriamicina. ${ }^{19}$

A fadiga foi o efeito colateral mais freqüente e problemático que persistiu durante a radioterapia em mulheres com câncer de mama no estudo realizado por Irvine. et al..$^{20} \mathrm{O}$ s outros estudos evidenciaram que a fadiga aumenta durante a radioterapia, e permanece com um platô entre a quarta e quinta semanas de radioterapia até a segunda semana após o término do tratamento. ${ }^{13,15}$

O s níveis de fadiga foram mais altos na última semana da radioterapia sendo que $53 \%$ das mulheres referiram estar mais fatigadas no final da radioterapia, $27 \%$ relataram que a fadiga estava no mesmo nível e $20 \%$ tinham menos fadiga. ${ }^{17,} 20 \mathrm{Em}$ geral a fadiga retorna ao nível pré-tratamento dois a três meses depois do término da radioterapia. ${ }^{13,15,20}$ Além disso, quanto maior a fadiga encontrada antes da radioterapia, maior será a fadiga durante a radioterapia. ${ }^{13}$

$\mathrm{N}$ ão foi encontrada nenhuma relação entre a fadiga e algumas variáveis como idade,20 estado conjugal, nível de educação $0^{10,18}$ e estádio da doença. 18,200 tipo de cirurgia esteve associado à fadiga em apenas um estudo..$^{10} \mathrm{Com}$ relação ao peso corporal Irvine et al. ${ }^{20}$ não encontraram relação entrea fadiga ea mudança de peso, porém Wratten et al. ${ }^{13}$ relatam que a fadiga está significativamente correlacionada com o aumento do índice de massa corpórea. Segundo Kumar et al. ${ }^{12}$ a quimioterapia influenciou a função tireoidiana contribuindo no aumento do peso, amenorréia, fadiga e diminuição da atividade física; esta última foi também um indicativo de redução nas horas trabalhadas. A fadiga interferiu em atividades como tomar banho, vestir-se, trabalhar, concentrar-se, relacionar-se com os outros, ter prazer da vida e no humor. ${ }^{18}$ Além disso, esteve correlacionada com a piora do nível de atividade física, ${ }^{16-18}$ com o performance status (PS) e fraqueza muscular. ${ }^{18}$ 
Q uadro1. Características dos estudos sobre fadiga em mulheres em tratamento para câncer de mama, janeiro de 1996 a setembro de 2004.

\begin{tabular}{|c|c|c|c|c|c|}
\hline Autor (ano) & $N$. & $\begin{array}{l}\text { Faixa etária } \\
\text { (anos) }\end{array}$ & Estádios & $\begin{array}{l}\text { Tratamento } \\
\text { adjuvante }\end{array}$ & Período entre as medidas \\
\hline Bennett et al. ${ }^{9}(2004)$ & 109 & $36-77$ & Ie II & QT e/ou RT & durante ou após QT \\
\hline Haghighat et al. ${ }^{10}(2003)$ & 112 & $22-76$ & I a IV & QT e RT & após $3^{\circ}$ ciclo de QT \\
\hline Lee $^{11}(2001)$ & 122 & $27-63$ & Ie II & QT e RT & durante QT ou RT \\
\hline Kumar et al. ${ }^{12}(2004)$ & 170 & $25-75$ & I a III B & QT & início e final da QT, após 6 meses de tratamento \\
\hline Wratten et al. ${ }^{13}$ (2004) & 52 & $31-74$ & - & RT & antes, 5 semanas durante RT, 2 e 6 semanas pós RT \\
\hline Berger et al. ${ }^{14}(2003)$ & 21 & $43-66$ & I e II & QT e RT & 1, 2 e 3 meses após QT, e 1 ano após o primeiro ciclo de QT \\
\hline Geinitz et al. ${ }^{15}$ (2001) & 41 & $34-77$ & | a || B & QT e RT & antes, fim da $1^{\mathrm{a}}$ e $5^{\mathrm{a}}$ semanas de RT, 2 meses pós RT \\
\hline $\begin{array}{l}\text { Berger e Higginbotham }{ }^{16} \\
(2000)\end{array}$ & 14 & $32-69$ & | e II & QT & $\begin{array}{l}48 \text { horas antes, e } 3 \text { medidas durante a } 3^{\mathrm{a}} \text { QT, um e dois meses } \\
\text { após a } 4^{\mathrm{a}} \text { QT. }\end{array}$ \\
\hline Jansen et al. ${ }^{17}(2000)$ & 46 & $28-77$ & Iniciais & RT & antes da RT e na última semana da RT \\
\hline $\begin{array}{l}\text { Jacobsen et al . }{ }^{18} \\
\text { (1999) }\end{array}$ & $\begin{array}{c}108 \\
\text { (controle:54 e } \\
\text { câncer:54) }\end{array}$ & $28-77$ & I a III & QT & antes do $1 \stackrel{\circ}{ }, 3^{\circ}$ e $4^{\circ}$ ciclos de QT \\
\hline Berger ${ }^{19}(1998)$ & 72 & $30-69$ & I e II & QT & $\begin{array}{l}48 \text { horas após } \circ 1^{\circ}, 2^{\circ} \text { e } 3^{\circ} \text { ciclos de QT, e no meio do período } \\
\text { entre os ciclos }\end{array}$ \\
\hline Irvine et al. ${ }^{20}$ (1998) & 76 & $33-81$ & Iniciais & RT & antes, $1^{\mathrm{a}}, 2^{\mathrm{a}}$ e última semana da $\mathrm{RT}$ e 3 e 6 meses pós RT \\
\hline Hoskin $^{21}$ (1997) & 93 & média 51,4 & - & QT e/ou RT, & 7 a 10 dias, 1, 2, 3e 6 meses, e 1 ano após cirurgia \\
\hline Longman et al. ${ }^{22}$ (1997) & 53 & $26-80$ & I a IV & QT, RT, HT & $\begin{array}{l}\text { início do tratamento, } 6 \text { a } 8 \text { semanas após QT ou RT e } 3 \text { meses } \\
\text { depois da } 2^{9} \text { medida }\end{array}$ \\
\hline
\end{tabular}

Abreviações: H T = hormonioterapia, Q T = quimioterapia, RT = radioterapia.

Q uadro2. Instrumentos e escores de fadiga empregados em mulheres com câncer de mama durante 0 tratamento, janeiro de 1996 a setembro de 2004.

\begin{tabular}{|c|c|c|c|c|}
\hline Autor (ano) & $\begin{array}{l}\text { Instrumento } \\
\text { utilizado }\end{array}$ & $\begin{array}{l}\text { Número } \\
\text { medidas }\end{array}$ & $\begin{array}{l}\text { Escore medido de fadiga } \\
\text { (variação da escala) }\end{array}$ & Prevalência de fadiga \\
\hline Bennett et al..$^{9}$ (2004) & $\begin{array}{l}\text { FACT F } \\
\text { SPHERE }\end{array}$ & 1 & 18,8 a $30,8(0-52)$ & $37 \%$ \\
\hline Haghighat et al. ${ }^{10}(2003)$ & CFS & 1 & $18,7(0-60)$ & $49 \%$ após $3^{\circ}$ ciclo \\
\hline Lee ${ }^{11}(2001)$ & PFS & 1 & $5,55(0-10)$ & 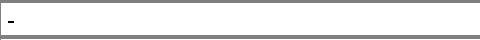 \\
\hline Kumar et al. ${ }^{12}(2004)$ & $\mathrm{FSI}$ & 3 & - & $42 \%$ no início e $94 \%$ ao final do tratamento \\
\hline Wratten et al. ${ }^{13}(2004)$ & FACT F & 8 & - & $43 \%$ durante a RT \\
\hline Berger et al. ${ }^{14}(2003)$ & PFS & 4 & 2,9 a $3,5(0-10)$ & $\begin{array}{l}29 \text { a } 43 \% \text { fadiga moderada para severa após } \\
\text { completar QT }\end{array}$ \\
\hline Geinitz et al. ${ }^{15}(2001)$ & $\begin{array}{l}\text { FAQ } \\
\text { VAS }\end{array}$ & 7 & $\begin{array}{l}15,3(0-50) \\
3,5(0-10) \text { durante a RT }\end{array}$ & - \\
\hline $\begin{array}{l}\text { Berger e Higginbotham }{ }^{16} \\
(2000)\end{array}$ & PFS & 6 & 3,6 a $5,6(0-10)$ & - \\
\hline Jansen et al. ${ }^{17}(2000)$ & $\begin{array}{l}\text { SF-36 } \\
\text { RSCL }\end{array}$ & 2 & - & $\begin{array}{l}53 \% \text { ao final da radioterapia } \\
51 \% \text { ao final da radioterapia }\end{array}$ \\
\hline Jacobsen et al. ${ }^{18}(1999)$ & $\begin{array}{l}\text { POMS-F } \\
\text { FSI } \\
\text { MSAS F }\end{array}$ & 3 & $\begin{array}{l}\text { câncer: } 9,90 ; 9,18 ; 11,06 \text { (0-28) POMS-F } \\
\text { controle: } 6,59 ; 6,83 ; 5,11(0-28) \text { POMS-F } \\
\text { câncer: } 4,03 ; 4,11 ; 4,13(0-10) \mathrm{FSI} \\
\text { controle: } 2,91 ; 3,30 ; 2,76(0-10) \mathrm{FSI}\end{array}$ & 72 a $94 \%$ (MSAS-F) \\
\hline Berger ${ }^{19}(1998)$ & PFS & 6 & 3,41 a $4,82(0-10)$ & $\begin{array}{l}\text { Intensidade moderada a alta em } 81-82-77 \% \\
\text { nas } 48 \mathrm{hs} \text {. } \\
71-62-57 \% \text { no meio dos ciclos }\end{array}$ \\
\hline Irvine et al. ${ }^{20}(1998)$ & PBFFC & 6 & 23,61 a $25,40(13-39)$ & - \\
\hline Hoskin $^{21}$ (1997) & TRI & 6 & - & 1 a $35 \%$ \\
\hline Longman et al. ${ }^{22}$ (1997) & SESC & 3 & - & 70 a $79 \%$ \\
\hline
\end{tabular}

Abreviações: CFS= Cancer Fatigue Scale, FACT-F = Functional Assessment of Cancer Therapy-Fatigue, FAQ = Fatigue Assessment Q uestionnaire, $\mathrm{FSI}=$ Fatigue Symptom Inventory, M SAS-F= M emorial Symptom Assessment Scale-Fatigue, PBFFC = Pearson Byards Fatigue Feeling Checklist, PFS= Piper Fatigue Scale, POM S-F= Profile of M ood States Fatigue Scale, RSCL= Rotterdam Symptom Check list, SESC = Side Effect Symptom Checklist, SF-36= Short-Form health status survey, SPH ERE = Somatic and Psychological H ealth Report, TRI=Treatment Recovery Inventory, $\mathrm{VAS}=\mathrm{Visual}$ Analogue Scale, QT = quimioterapia, $\mathrm{RT}=$ radioterapia.

Pacientes com depressão, ansiedade, estresse, problemas psicológicos e emocionais apresentaram mais fadiga do que aqueles sem esses sintomas em quatro estudos. ${ }^{9,10,18,20} \mathrm{O}$ utro estudo ${ }^{15}$ não encontrou evidências de que a ansiedade, depressão, níveis séricos de interleucina 1 beta, interleucina 6, fator de necrose tumoral alfa ou níveis de hemoglobina estivessem associadas à fadiga. ${ }^{15}$ Lee $^{11}$ encontrou pequena, mas significante relação entre fadiga e falta de esperança, que pode ser atribuída à falta de energia. Em relação à qualidade de vida a fadiga apresentou um impacto negativo. ${ }^{17,20}$ 
Em relação ao sono, a insônia foi associada a maior fadiga em pacientes submetidas a quimioterapia em 3 estudos. ${ }^{14,16,18}$ Também foi demonstrada uma associação entre a fadiga e a dor em dois estudos. ${ }^{10,18}$

\section{DISCUSSÃO}

Estudos avaliados nessa revisão mostram que a fadiga é comum em pacientes com câncer de mama e aumenta significativamente durante a quimioterapia e radioterapia. A forma como a radioterapia e a quimioterapia causam a fadiga ainda não está clara. ${ }^{7}$

$\mathrm{H}$ á diversos instrumentos para avaliar a fadiga em pacientes com câncer e que apresentam pontuações e escores variados. ${ }^{23,24}$ As variações dos escores de fadiga encontrados nos estudos avaliados podem ser devido ao período em que a fadiga foi medida, que foi desde 0 período anterior ao tratamento até um ano após a cirurgia. Entretanto a fadiga tem sido mais comum na última semana de radioterapia, durante os ciclos de quimioterapia e principalmente nos primeiros dias após a quimioterapia nos estudos dessa revisão de literatura. Q uanto maior a fadiga encontrada antes da radioterapia, maior será a fadiga durante a radioterapia. ${ }^{13}$

Algumas limitações foram encontradas com relação aos estudos avaliados. O s estudos transversais são restritos nas conclusões sobre a mudança no grau de fadiga durante o tratamento ou seguido ao término do tratamento. M etodologicamente os melhores estudos são os longitudinais, nos quais a paciente é avaliada em múltiplas ocasiões duranteo tratamento e permitem mais informações sobre a história natural da fadiga em pacientes com câncer de mama.

A fadiga tem um grande impacto sobre a qualidade de vida em pacientes oncológicos. ${ }^{25} \mathrm{~A}$ equipe de saúde deve avaliar a fadiga cuidadosamente e deve orientar as mulheres sobre sua ocorrência antes da radioterapia e quimioterapia, e também disponibilizar às pacientes estratégias de como intervir na fadiga. As abordagens devem incluir intervenções farmacológicas com antidepressivos, psicoestimulantes, baixas doses de corticosteóides; e intervenções não farmacológicas como exercícios físicos, nutrição adequada, manejo do estresse e terapia cognitiva, modificação dos padrões de atividade, repouso e sono. ${ }^{26} \mathrm{H}$ á evidências de que muitas mulheres ainda continuam com fadiga depois do tratamento, e a fadiga severa está associada com fatores físicos, psicológicos, sociais, cognitivos e comportamentais que afetam a vida diária. ${ }^{25,27}$

Ainda há muito para ser descoberto em relação à fadiga tais como mecanismos causadores, diferenças individuais apesar de receberem o mesmo tratamento, prevalência em populações específicas de câncer e também quanto às intervenções para preveni-la ou aliviála.

$\mathrm{N}$ ão há instrumentos para avaliar a fadiga em pacientes com câncer validados em língua portuguesa, o que indica a necessidade de se construir ou validar para essa finalidade. ${ }^{23} \mathrm{D}$ evem ser realizadas mais pesquisas controladas, inclusive em mulheres brasileiras com câncer de mama com grupos mais homogêneos, instrumentos válidos e confiáveis.

\section{REFERÊNCIAS}

1. Instituto $\mathrm{N}$ acional de $\mathrm{C}$ âncer. Estimativas de incidênciae mortalidadepor câncer no Brasil. Rio de J aneiro (Brasil): INCA; 2003.

2. De Jong N, Courtens AM, Abu-Saad, Schouten HC. Fatigue in patients with breast cancer receiving adjuvant chemotherapy: a review of the literature. Cancer $\mathrm{N}$ urs. 2002;25(4):283-97.

3. Servaes $P$, Verhagen $S$, Bleijenber $G$. Fatigue in cancer patients during and after treatment: prevalence, correlates and interventions. Eur J Cancer. 2002;38:27-43.

4. O Idervoll LM , K aasaS, H jermstad M J, Lund JA, LogeJH . Physical exercise results in the improved subjective wellbeing of a few or is effective rehabilitation for all cancer patients? Eur J Cancer. 2004;40(7):951-62.

5. N ational $C$ ancer Institute [homepage on the Internet]. U nited States: $\mathrm{N}$ ational Institutes of $\mathrm{H}$ ealth [cited 2004 0 ct 20]. Fatiga (PDQ $\left.{ }^{\circledR}\right)$ : descripcion. Available from: http://www.nci.nih.gov/espanol/pdq/cuidados-medicosapoyo/fatiga/healthprofessional/allpages

6. Magnusson $\mathrm{K}$, M oller $\mathrm{A}$, Ekman $\mathrm{T}$, Wallgren $\mathrm{A}$. A qualitative study to explore the experience of fatigue in cancer patients. Eur J C ancer C are. 1999;8:224-32.

7. Barnes $E A$, Bruera $A$. Fatigue in patients with advanced cancer: a review. Int] Gynecol C ancer. 2002;12:424-8.

8. Jacobsen PB, Andrykowski M A, Thors CL. Relationship of catastrophizing to fatigue among women receiving treatment for breast cancer. J C onsult C lin Psychol. 2004 Apr;72(2):355-61.

9. Bennett B, Goldstein D, Lloyd A, D avenport T, H ickiel. Fatigue and psychological distress- exploring the relationship in women treated for breast cancer. Eur J Cancer. 2004;40:1689-95.

10. $\mathrm{H}$ aghighat $\mathrm{S}, \mathrm{Akbari} M E, H$ olakouei $\mathrm{K}$, Rahimi $A$, $M$ ontazeri $A$. Factors predicting fatigue in breast cancer patients. Support C are C ancer. 2003;11(8):533-8.

11. LeeEH . Fatigue and hope: relationships to psychosocial adjustment in Korean women with breast cancer. Appl N urs Res. 2001;14(2):87-93.

12. Kumar N, Allen KA, Riccardi D, Bercu BB, Cantor A, 
Minton S, et al. Fatigue, weight gain, lethargy and amenorrhea in breast cancer patients on chemotherapy: is subclinical hypothyroidism the cul prit? B reast $C$ ancer Res Treat. 2004;83(2):149-59.

13. Wratten C, Kilmurray J, N ash S, Seldon M , H amilton CS, $O$ 'Brien $P C$, et al. Fatigueduring breast radiotherapy and itsrelationship to biological factors. Int J Radiat O ncol Biol Phys. 2004;59(1):160-7.

14. Berger AM, VonEssen S, Kuhn BR, Piper BF, Agrawal S, Lynch JC, et al. Adherence, sleep, and fatigue outcomes after adjuvant breast cancer chemotherapy: results of a feasibility intervention study. O ncol Nurs Forum. 2003;30(3):513-22.

15. Geinitz H, Zimmermann FB, Stoll P, Thamm R, Kaffenberger W, Ansorg K, et al. Fatigue, serum cytokine levels, and blood cell countsduring radiotherapy of patients with breast cancer. Int J Radiat O ncol Biol Phys. 2001;N ov 1;51(3):691-8.

16. Berger AM , H igginbotham P. C orrelates of fatigueduring and following adjuvant breast cancer chemotherapy: a pilot study. O ncol N urs Forum. 2000;27(9):1443-8.

17. J ansen SJ, StiggelboutAM , N ooij M A, N oordijkEM , Kievit $J$. Response shift in quality of life measurement in earlystage breast cancer patients undergoing radiotherapy. Q ual Life Res. 2000;9(6):603-15.

18. Jacobsen PB, H ann D M , Azzarello LM , H orton J, Balducci $\mathrm{L}$, Lyman $\mathrm{GH}$. Fatigue in women receiving adjuvant chemotherapy for breast cancer: characteristics, course, and correlates. J Pain Symptom M anage. 1999;18(4):233-42.

19. Berger AM . Patterns of fatigueand activity and rest during adjuvant breast cancer chemotherapy. 0 ncol N urs Forum. 1998;25(1):51-62.

20. Irvine D M , Vincent L, Graydon JE, Bubela N . Fatiguein women with breast cancer receiving radiation therapy. C ancer N urs. 1998;21(2):127-35.

21. H oskins $C N$. B reast cancer treatment-related patterns in side effects, psychological distress, and perceived health status. O ncol N urs Forum. 1997;24(9):1575-83.

22. Longman AJ, Braden CJ, M ishel M H . Pattern of association over time of side-effects burden, self-help, and self-carein women with breast cancer. O ncol Nurs Forum. 1997;24(9):1555-60.

23. M otaD D CF. Instrumentos deauto-relato para avaliação de fadiga: umarevisão sistemática [dissertação]. São Paulo (SP): EscoladeEnfermagem daU niversidadedeSão Paulo; 2003.

24. SchwartzAH . Validity of cancer-related fatigueinstruments. Pharmacotherapy. 2002;22(11):1433-41.

25. Curt GA. Impact of fatigue on quality of lifein oncology patients. Semin H ematol. 2000;37 Suppl 6:14-7.

26. Portenoy RK, Itri LM . Cancer related fatigue: guidelinesfor evaluation and management. O ncologist. 1999;4:1-10.

27. Servaes $P$, Verhagen $S$, Bleijenberg $G$. D eterminants of chronic fatiguein disease freebreast cancer patients: acrosssectional study. Ann O ncol. 2002;13:589-98. 